\title{
ARTICLE Realization of palladium-based optomechanical cantilever hydrogen sensor
}

\author{
Steven J. McKeown, Xiaozhen Wang, Xin Yu and Lynford L. Goddard
}

Hydrogen has attracted attention as an alternative fuel source and as an energy storage medium. However, the flammability of hydrogen at low concentrations makes it a safety concern. Thus, gas concentration measurements are a vital safety issue. Here we present the experimental realization of a palladium thin film cantilever optomechanical hydrogen gas sensor. We measured the instantaneous shape of the cantilever to nanometer-level accuracy using diffraction phase microscopy. Thus, we were able to quantify changes in the curvature of the cantilever as a function of hydrogen concentration and observed that the sensor's minimum detection limit was well below the 250 p.p.m. limit of our test equipment. Using the change in curvature versus the hydrogen curve for calibration, we accurately determined the hydrogen concentrations for a random sequence of exposures. In addition, we calculated the change in film stress as a function of hydrogen concentration and observed a greater sensitivity at lower concentrations.

Keywords: hydrogen detection; imaging and sensing; interference microscopy; optomechanics; optical sensors; surface dynamics Microsystems \& Nanoengineering (2017) 3, 16087; doi:10.1038/micronano.2016.87; Published online: 27 March 2017

\section{INTRODUCTION}

Hydrogen has always been viewed as a promising alternative to fossil fuels, and it can also function as an effective energy storage medium for intermittent energy sources. In addition, hydrogen is used in a range of other industries, including chemical production, metal refining, and food processing. A major safety concern with hydrogen is combustibility. Therefore, early leak detection and concentration determination of hydrogen have been areas of intense research ${ }^{1-3}$. There are various types of hydrogen sensors that use a wide range of detection mechanisms. Lundström et al. ${ }^{4}$ proposed metal oxide semiconductor (MOS)-type hydrogen sensors ${ }^{5}$. However, MOS sensors suffer from drawbacks such as premature saturation of detectable hydrogen concentrations and low sensitivity. Other MOS-based devices have been used as hydrogen sensors, such as MOS field-effect transistors (FETs) ${ }^{6,7}$, high electron mobility transistors ${ }^{8-10}$, and Schottky diode-type FETs ${ }^{11,12}$ However, these devices require complicated fabrication processes and have high production costs. Optical gas sensors ${ }^{13-21}$ not only overcome these disadvantages but also have other unique advantages, such as negligible electrical interference, no risk of ignition from an electrical spark, and the ability to work at high temperatures or in harsh environments.

Palladium (Pd) can absorb up to 900 times its own weight in hydrogen gas at room temperature ${ }^{22}$. Compared with platinum ${ }^{23}$, $\mathrm{Pd}$ film is more popular because of its lower cost. Pd alloys with $\mathrm{Ag}, \mathrm{Au}, \mathrm{Ni}$, and $\mathrm{WO}_{3}$ have also been studied ${ }^{24-29}$ because of their improved response time ${ }^{24,25,27}$, sensitivity ${ }^{28,29}$, and durability ${ }^{26}$ as a sensing material. The alloys can avoid blistering effects ${ }^{26}$ and the $a$ to $\beta$ phase transition at higher hydrogen concentrations ${ }^{25,27}$.
During the adsorption process, $\mathrm{Pd}$ hydride $(\mathrm{PdH})$ is formed and the optical and mechanical properties of the $\mathrm{Pd}$ film are changed ${ }^{24,30} . \mathrm{PdH}$ can exist in the pure $a$ phase, the mixed $a+\beta$ phase, or the pure $\beta$ phase when the ambient hydrogen concentration is low, medium, or high, respectively. The phase transition points can occur anywhere from 0.1 to $2 \%$ hydrogen depending on the thickness, quality, and structure of the film ${ }^{30-32}$. When the concentration is below the transition point for the pure $\beta$ phase, the optical and mechanical properties of the Pd film will recover after hydrogen removal ${ }^{13,14}$. This property makes a Pd film an effective functional layer for optical hydrogen detection ${ }^{3,31,33}$. $\mathrm{Pd}$-coated cantilevers were previously studied as capacitive hydrogen sensors ${ }^{34-36}$. In addition to changes in reflectivity, stress induced by hydrogen-induced lattice expansion (HILE) causes the cantilever to deform. Our group previously measured HILE in Pd microdiscs ${ }^{37}$.

Here we fabricated nanoscale Pd-coated cantilevers and characterized the devices using diffraction phase microscopy $(D P M)^{37-43}$. The DPM technique achieves real-time single-shot non-destructive quantitative phase imaging in situ with nanometer-level sensitivity. The novelty of this work is the sensing modality because we can measure the instantaneous threedimensional shape of the cantilever and use it to determine the change in curvature of the $\mathrm{Pd}$ film as a function of $\mathrm{H}_{2}$ concentration. Furthermore, we successfully demonstrated that random hydrogen concentrations can be accurately determined using the measured curvature changes of the Pd film cantilever. In addition, we used the curvature data to extract the change in residual stress of the Pd film as a function of hydrogen concentration. 


\section{MATERIALS AND METHODS}

Several cantilever geometries were simulated based on previous measurements ${ }^{37}$ on the expansion of Pd films. The important physical parameters are the cantilever's beam material, film thicknesses, and length ${ }^{44}$. A process flow for the fabrication can be seen in Figure 1. Cantilevers were fabricated on $\langle 100\rangle$ silicon using an anisotropic $\mathrm{KOH}$ etch process, with the $\langle 111\rangle$ plane forming the sidewalls of the cavity under the cantilever. First, the silicon nitride $\left(\mathrm{SiN}_{x}\right)$ cantilever layer was deposited via plasmaenhanced chemical vapor deposition (PECVD) using a Mesc Multiplex PECVD tool (Surface Technology Systems (STS), Stratham, NH, USA). After calibrating the deposition rate on a dummy sample, the deposition time was computed and used to obtain the desired nitride thickness on the real sample. Silicon nitride was selected as the beam material because of its compatibility with the $\mathrm{KOH}$-based fabrication process and because the intrinsic stress in the film can be minimized by tuning the deposition conditions. Specifically, we used mixed RF frequencies of 13.56 and $380 \mathrm{kHz}$ to minimize the intrinsic film stress. At this point, the intrinsic film stress was measured to be $+190 \mathrm{MPa}$ using a 500TC stress measurement tool (Frontier Semiconductor Inc., San Jose, CA, USA); the thickness was confirmed to be $1010 \mathrm{~nm}$ using an LSEUSB ellipsometer (Gaertner Scientific Corporation, Skokie, IL, USA). Next, contact photolithography was used to define C-shaped etch windows that were etched through the $\mathrm{SiN}_{x}$ layer using reactive ion etching (RIE) in a PlasmaLab $\mu \mathrm{P}$ Freon RIE tool (Plasma Technology, defunct). The size and spacing of the windows were varied to fabricate an array of cantilevers with different sizes. Finally, undercutting of the cantilevers was achieved through convex corner etching using a $20 \%$ aqueous $\mathrm{KOH}$ etchant at $80^{\circ} \mathrm{C}$. The lateral undercut etch process is self-limiting; nevertheless, the etching was stopped once the cantilevers were fully undercut to avoid thinning the wafer. Next, we used a CHA SEC-600 e-beam evaporator (CHA Industries Inc., Fremont, CA, USA) to deposit $1.5 \mathrm{~nm}$ of $\mathrm{Cr}$ and $1.5 \mathrm{~nm}$ of $\mathrm{Ni}$ to form an adhesion layer followed by the $\mathrm{Pd}$ sensing layer of the desired thickness on each sample. Thicknesses were precisely controlled during deposition using a crystal monitor. The deposition rates were 1.5 and $2 \AA^{-1} s^{-1}$ for the $\mathrm{Cr} / \mathrm{Ni}$ adhesion layer and the $\mathrm{Pd}$ sensing layer, respectively. The primary constraints on the Pd and nitride thicknesses were given by mechanical failure points and the numerical aperture of the measurement system. Very thin and long cantilevers would potentially create excellent sensors because the hydrogeninduced deflections would be large. However, in both these extremes, the cantilevers could more easily break. Additionally, to characterize accurately the structures, we need to ensure that the reflected light is captured by the objective of the DPM system. The latter effect was the primary limitation on the minimum nitride thickness and on the maximum cantilever length. A nitride

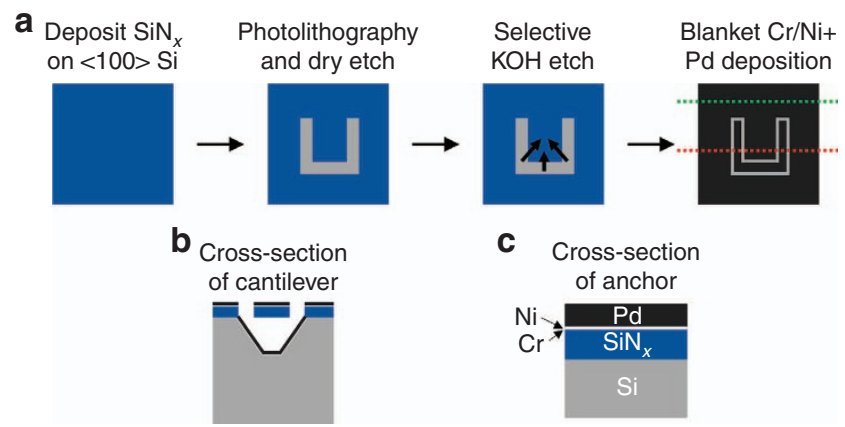

Figure 1 (a) Fabrication process flow. Cross-section of (b) the cantilever and (c) the anchor, through the red and green dotted lines, respectively, in the last image of (a). Pd, palladium. thickness of $1010 \mathrm{~nm}$ was selected. The Pd layer was selected to be optically thick to ensure that multiple reflections did not complicate the interpretation of the DPM phase images. To study the dependence of sensor performance on Pd film thickness, two batches of devices were fabricated: one with a Pd layer thickness of $25 \mathrm{~nm}$ and the other with a Pd layer of $50 \mathrm{~nm}$. Arrays of cantilevers were fabricated on both wafers with a variety of lengths and widths to study the dependence of sensor performance on cantilever size.

For testing, the samples were mounted in a machined aluminum test chamber. The chamber had interior dimensions of $2.5 \times 1.7 \times 0.64 \mathrm{~cm}$, resulting in a volume of $2.7 \mathrm{~cm}^{3}$. The chamber had two lateral gas inlet ports located on the sides and an outlet port situated directly over the sample. The outlet port was a small hole machined through the chamber window. The hole reduces noise in the DPM measurement because it allows light to pass through without any reflection off of the glass window. Two mass flow controllers (MFCs) were used to control the hydrogen concentration and keep the chamber at a slight positive pressure. In addition, solenoid valves were also used in series with each MFC to ensure that no gas was flowing when they were set to zero flow rate. Two tubes, each having an inner diameter of $1 / 8$ in, connected the solenoid valves to a common tube for gas mixing before splitting back to connect to the two lateral gas inlet ports of the chamber. The total tube length was $8 \mathrm{in}$, resulting in an additional volume of $1.6 \mathrm{~cm}^{3}$. The MFCs and solenoid valves were controlled through LabVIEW. One MFC controlled the flow rate of an already prediluted hydrogen mixture, while the other MFC set the flow rate for air, which served as the diluent. The combined flow rate was $200 \mathrm{sccm}$, which is small enough to ensure laminar flow in the chamber. Two different concentrations of diluted hydrogen were used to achieve the desired concentrations: 4\% hydrogen in nitrogen (SJ Smith Co., Davenport, IA, USA; UC3344) for the [0.2\%, 1\%] hydrogen experiments and $0.2 \%$ hydrogen in air (Airgas Inc., Radnor Township, PA, USA; X02AI99C30053D2) for the [250 p.p.m., 0.2\%] hydrogen experiments. The testing times varied, but a standard measurement consisted of exposure times of $30 \mathrm{~min}$ for both the test concentration and the subsequent recovery in air. All of the gas flow was automated via LabVIEW and was synced to the time stamp on the DPM images for analysis. For these measurements, a $405 \mathrm{~nm}$ laser (Thorlabs Inc., Newton, NJ, USA; S3FC405) was used as the illumination source.

\section{RESULTS}

Figure $2 \mathrm{a}$ shows the undercutting process used to fabricate the suspended structure at various points in time. Figure $2 b$ shows the undercut etch distance versus time. Scanning electron microscope (SEM) images of one of the final structures after Pd deposition can be seen in Figures $2 \mathrm{c}$ and $\mathrm{d}$. Although not intentional, the sloped bottom of the cavity as a result of the undercutting process is beneficial in that it prevents reflections from the cavity from interfering with the measurement in the case of partially transparent Pd films. SEM imaging also showed a slight initial upward curvature to the cantilevers that was later confirmed with DPM. Measurements of the film stress for the nitride showed that the layer was compressively strained, with a measured stress value of $\sim+190 \mathrm{MPa}$. This curvature was largely independent of the $\mathrm{Pd}$ film, implying that it is a result of a stress gradient in the nitride and is not because of relaxation kinetics of the final bimorph structure after fabrication. Because the stress in the deposited nitride film decreases as distance from the substrate increases, the top is more tensile than the bottom, resulting in upward curvature after undercutting.

The topographic profile of the entire cantilever can be measured using DPM. Compared with point or bulk area measurements, such as reflected or transmitted power spectra 
a

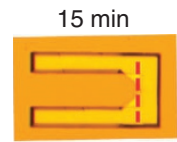

$60 \mathrm{~min}$

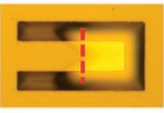

$90 \mathrm{~min}$

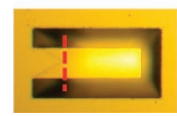

C

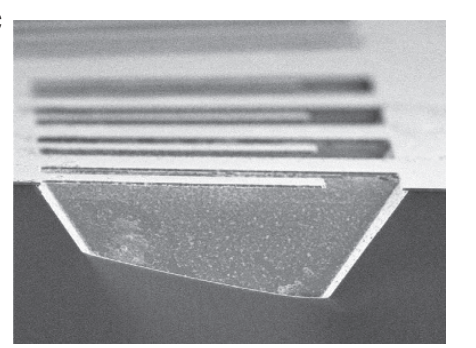

$30 \mathrm{~min}$

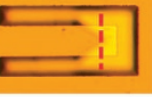

$75 \mathrm{~min}$

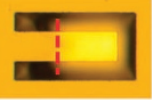

$105 \mathrm{~min}$

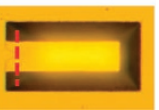

(n) b

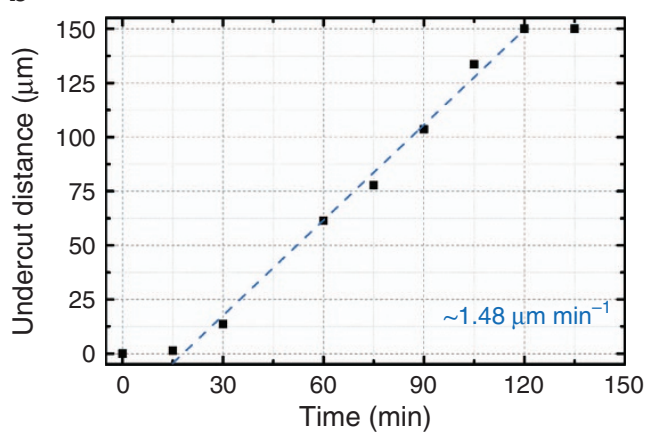

d

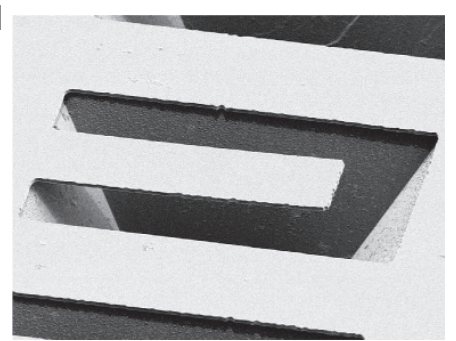

Figure 2 (a) Optical microscope images of the cantilever undercutting process at various points in time. (b) Undercut etch distance versus time. Scanning electron microscope (SEM) images of (c) cross-section view and (d) isometric view of a final fabricated cantilever.

a
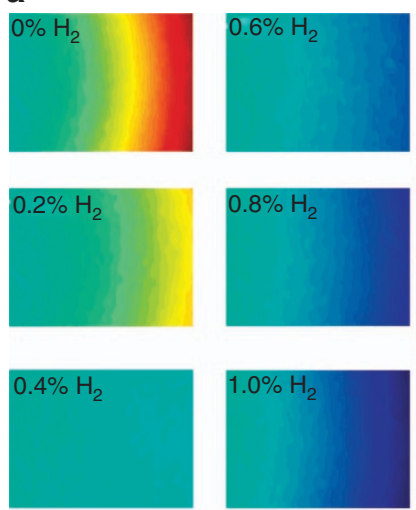

$20 \mu \mathrm{m}$

$$
\text { (1) }
$$



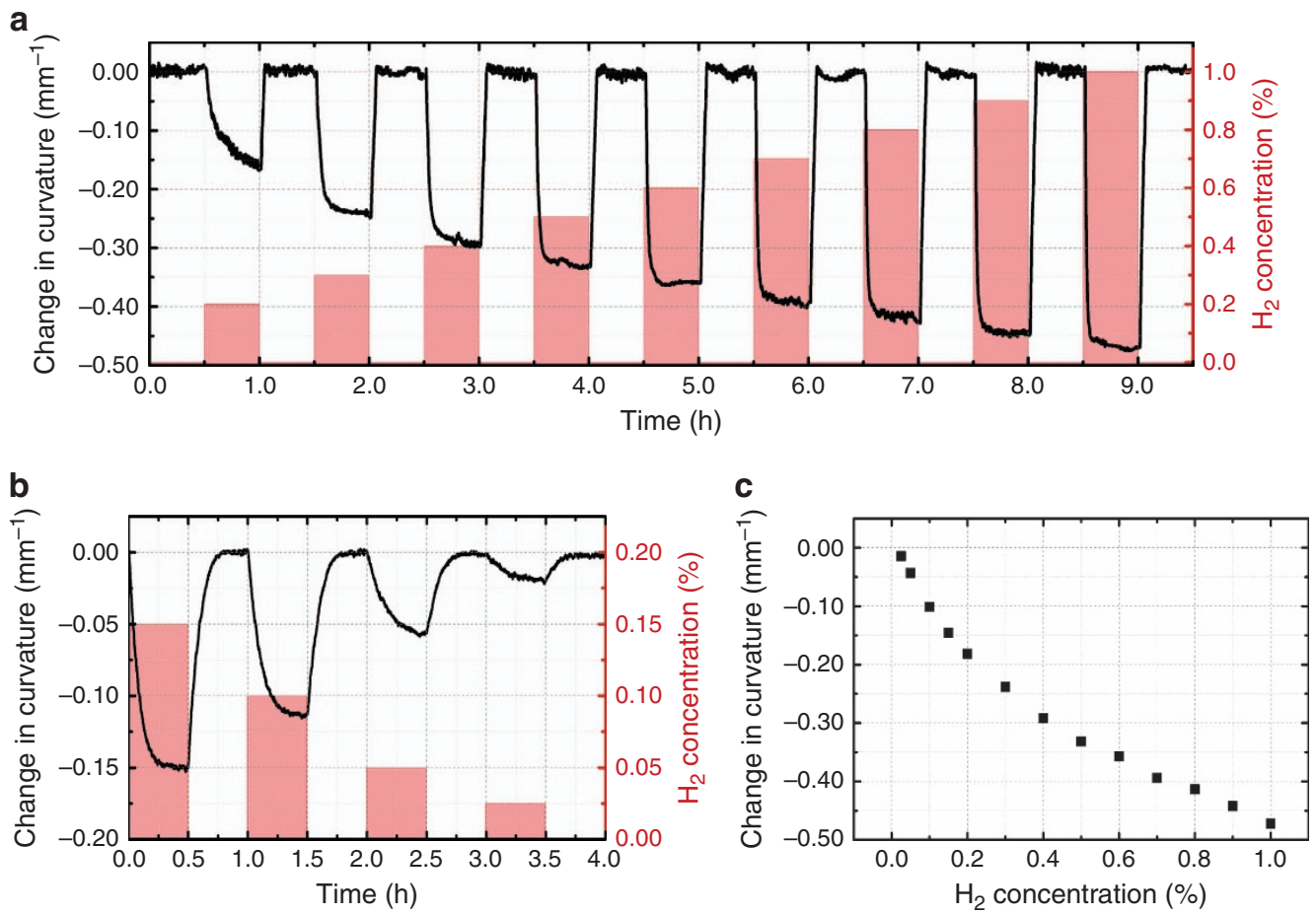

Figure 4 (a and $\mathbf{b}$ ) Temporal response of change in curvature to a pulse train of $\mathrm{H}_{2}$ at two sets of hydrogen concentrations of [0.2\%, 1.0\%] and [250 p.p.m., 0.2\%], respectively. Dimensions of the cantilever: $40 \times 70 \mu \mathrm{m}, h_{\mathrm{Pd}}=50 \mathrm{~nm}$ and $h_{\mathrm{s}}=1010 \mathrm{~nm}$. (c) The measured change in curvature

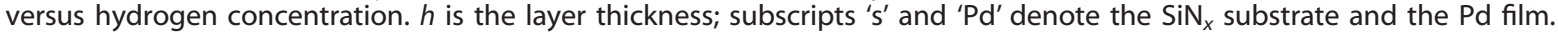
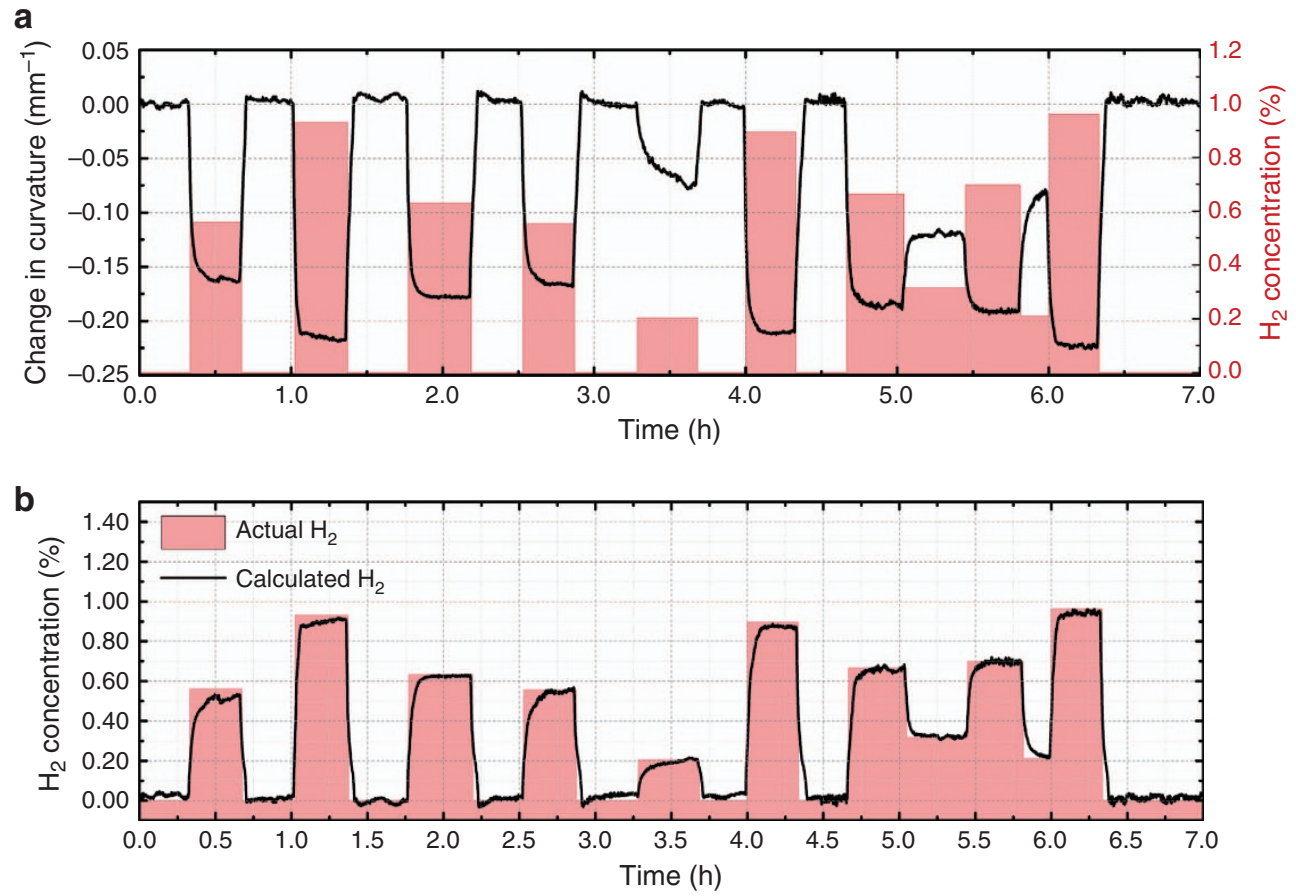

Figure 5 (a) Temporal response of the measured change in curvature for a random pulse train of $\mathrm{H}_{2}$. Dimensions of the cantilever: $40 \times 70 \mu \mathrm{m}$, $h_{\mathrm{Pd}}=50 \mathrm{~nm}$ and $h_{\mathrm{s}}=1010 \mathrm{~nm}$. (b) Calculated $\mathrm{H}_{2}$ concentration using the measured change in curvature and the calibration data shown in Figure 4c. $h$ is the layer thickness; subscripts ' $s$ ' and 'Pd' denote the $\mathrm{SiN}_{x}$ substrate and the Pd film.

Based on experiments and physical models, it can be shown ${ }^{45}$ that the deflection of a stressed cantilever as a function of position $x$ can be approximated by a quadratic

$$
z \simeq \frac{1}{2 R} x^{2}+\theta x+z_{0}
$$

where $R$ is the radius of curvature, $z_{0}$ is the initial height, and $\theta$ represents the initial angle at the anchor point caused by stresses. By fitting a measured cross-section in Figure $3 \mathrm{~b}$ to a quadratic function, the second-degree coefficient can be used to calculate the curvature $\kappa=1 / R$. In addition to the topographic information, the in situ DPM measurement allows for the collection of temporal 

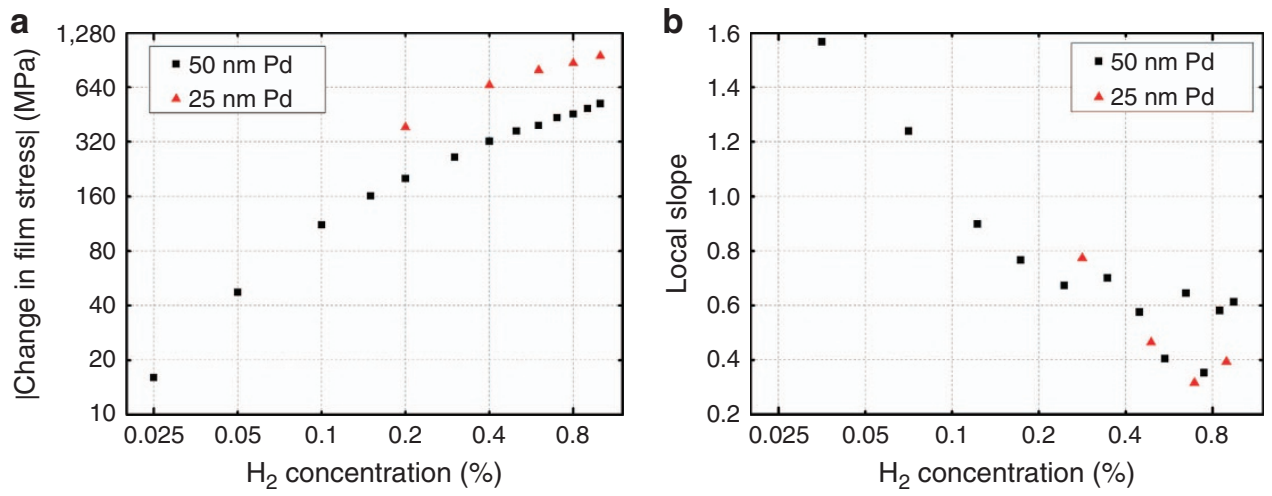

Figure 6 (a) The absolute value of the calculated change in film stress versus hydrogen concentration on a log-log scale. Dimensions of the cantilevers: $40 \times 70 \mu \mathrm{m}, h_{\mathrm{s}}=1010 \mathrm{~nm}$, with $h_{\mathrm{Pd}}=50$ and $25 \mathrm{~nm}$, respectively. (b) The local slope of (a) versus hydrogen concentration on a lin-log scale. $h$ is the layer thickness; subscripts ' $s$ ' and 'Pd' denote the $\mathrm{SiN}_{x}$ substrate and the Pd film.

data. By calculating the cross-section profile at each time step and fitting to a quadratic, the curvature, as a function of time, can be extracted during hydrogen exposure. These results are shown in Figures $4 a$ and $b$ for two different sets of test concentrations. Figure $4 c$ contains a summary of the curvature change. By using the curvature rather than a point deflection, data from the entire length of the cantilever are used, increasing the accuracy of the sensor. It can also be shown that the change in curvature is directly related to HILE. Hence, sensor to sensor variations, such as angle at the contact point, initial height or initial curvature, can be removed by considering the change in curvature rather than the $z$-position of a point on the cantilever. The curve in Figure $4 c$ was inverted and used to calibrate the sensor for the subsequent experiment.

A set of random concentrations was selected to test the predictability of the sensor for both return to zero measurements, as well as sequential random concentrations. The duration of each pulse was limited to a minimum of $20 \mathrm{~min}$ so that the sensor had time to fully respond. However, given that the $10-90 \%$ response times are on the order of $5 \mathrm{~min}$, shorter pulses could have been used with the introduction of slightly more error. The measured curvature for the random pulse test can be seen in Figure $5 \mathrm{a}$. Using the calibration, the ambient $\mathrm{H}_{2}$ concentration was calculated using the measured curvature. These results are shown in Figure $5 \mathrm{~b}$. There is a very small offset to the response at $0 \%$ that occurs when recovering from hydrogen exposure in that the cantilever returns to a state with compressive stress in the Pd layer resulting in more upward deflection. After some time, depending on the concentration, this is released and the baseline returns to normal. The error in the calculated hydrogen concentration has a value between 0 and $-0.04 \%$; that is, the determined hydrogen concentration is always less than the actual value. The error is relatively large compared with the noise in the measured height and curvature values; these factors alone correspond to an uncertainty of $0.003 \% \mathrm{H}_{2}$ for this cantilever. Additionally, although the applied $\mathrm{H}_{2}$ concentrations for pulses 1 , $3,4,7$, and 8 in Figure $5 b$ were almost the same, the pulse shapes for the recovered hydrogen concentration were different. These observations suggest systemic sources of error such as the memory effect of the Pd film that was discussed previously, which is most likely the result of the chemistry and thermodynamics of the film.

\section{DISCUSSION}

Here, the Pd layer is in the thin film regime, so we can use Stoney's equation ${ }^{46,47}$ to calculate the change in the residual film stress, $\Delta \sigma_{\mathrm{Pd}}$

$$
\Delta \sigma_{\mathrm{Pd}}(H)=\frac{E_{\mathrm{s}} h_{\mathrm{s}}^{2}}{6 h_{\mathrm{Pd}}\left(1-v_{\mathrm{s}}\right)} \Delta \kappa(H)
$$

where $\Delta K(H)$ is the curvature change of the cantilever. $H$ represents the hydrogen concentration, $E$ is Young's modulus, $h$ is the layer thickness, and $u$ is Poisson's ratio. The subscripts ' $s$ ' and 'Pd' denote the $\mathrm{SiN}_{x}$ substrate and the Pd film, respectively. Here, the $\mathrm{SiN}_{x}$ layer has a Young's modulus of $E_{\mathrm{s}}=250 \mathrm{GPa}$ and $\mathrm{a}$ Poisson's ratio of $v_{s}=0.23$ (Ref. 48).

The absolute value of the calculated change in film stress, $\left|\Delta \sigma_{\mathrm{Pd}}(H)\right|$, is plotted versus hydrogen concentration on a log-log scale in Figure 6a. Data are not available for the $25 \mathrm{~nm}$ sample at the lower concentrations because the sample was damaged while attempting a different experiment. In the $[0.2 \%, 1.0 \%]$ range, the change in the residual stress for the $25 \mathrm{~nm} \mathrm{Pd}$ film is approximately double that for the $50 \mathrm{~nm}$ film. This result is to be expected if one assumes that the linear expansion coefficient is the same for the two films.

The log-log plot shown in Figure $6 a$ is not linear with respect to hydrogen concentration over the full measurement range. To quantify the variation in slope, we define the local slope, $m$, as:

$$
m=\frac{\partial \ln \left|\Delta \sigma_{\mathrm{Pd}}(H)\right|}{\partial \ln H}
$$

and use the central finite difference to approximate this derivative. The results are plotted in Figure $6 \mathrm{~b}$. The local slope begins much higher than 1.0 at very low concentrations and decreases to values between 0.35 and 0.65 at higher concentrations. We attribute this decrease to the transition from the pure $a$ phase to the mixed $a+\beta$ phase because the lattice expansion dynamics are known to be different in the two phases ${ }^{31}$. To some extent, the local slope is a measure of the cantilever's sensitivity to hydrogen. Thus, we observe greater sensitivity at very low hydrogen concentrations.

\section{CONCLUSION}

In conclusion, we have shown that by measuring the instantaneous height profile of a $\mathrm{Pd}$ thin film cantilever to nanometer-level accuracy using DPM, we can quantify small changes in curvature and thereby determine the ambient hydrogen concentration. This optomechanical hydrogen gas sensor has both a low minimum detection limit as well as the ability to measure accurately the concentrations of a random sequence of hydrogen exposures. Further, the effect of hydrogen-induced lattice expansion on the residual stress in the $\mathrm{Pd}$ film was also determined. Understanding the nanoscale dynamics of $\mathrm{Pd}$ and other thin film materials is essential in the design of future optomechanical devices and novel 
sensor structures. We anticipate that the results and techniques presented in this paper will stimulate further research in these areas.

\section{ACKNOWLEDGEMENTS}

This work was supported by NSF Grant ECCS-0901388 (to SM and LLG) and a gift award (CG 587589) from Cisco Systems Inc. (to XW, XY, and LLG).

\section{COMPETING INTERESTS}

The authors declare no conflict of interest.

\section{REFERENCES}

1 Christofides C, Mandelis A. Solid-state sensors for trace hydrogen gas detection. Journal of Applied Physics 1990; 68: R1-R30.

2 Hübert T, Boon-Brett L, Black $G$ et al. Hydrogen sensors-a review. Sensors and Actuators B: Chemical 2011; 157: 329-352.

3 Silva SF, Coelho L, Frazao O et al. A review of palladium-based fiber-optic sensors for molecular hydrogen detection. IEEE Sensors Journal 2012; 12: 93-102.

4 Lundström I, Shivaraman S, Svensson C et al. A hydrogen-sensitive MOS fieldeffect transistor. Applied Physics Letters 1975; 26: 55-57.

5 Johansson M, Lundström I, Ekedahl LG. Bridging the pressure gap for palladium metal-insulator-semiconductor hydrogen sensors in oxygen containing environments. Journal of Applied Physics 1998; 84: 44-51.

$6 \mathrm{Kim} \mathrm{BJ}$, Yoon JH, Kim JS. Gas sensing characteristics of low-powered dual MOS-FET hydrogen sensors. Materials Chemistry and Physics 2013; 142: 594-599.

7 Safari M, Gholizadeh M, Salehi A. Modeling and simulation of a MOSFET gas sensor with platinum gate for hydrogen gas detection. Sensors and Actuators $B$ : Chemical 2009; 141: 1-6.

8 Cheng CC, Tsai YY, Lin KW et al. Characteristics of a Pd-oxide- $\mathrm{In}_{0.49} \mathrm{Ga}_{0.51} \mathrm{P}$ high electron mobility transistor (HEMT)-based hydrogen sensor. Sensors and Actuators B: Chemical 2006; 113: 29-35.

9 Hung CW, Lin $\mathrm{HL}$, Chen $\mathrm{Hl}$ et al. Comprehensive study of a Pd-GaAs high electron mobility transistor (HEMT)-based hydrogen sensor. Sensors and Actuators B: Chemical 2007; 122: 81-88.

$10 \mathrm{Yu}$ X, Li C, Low ZN et al. Wireless hydrogen sensor network using AlGaN/GaN high electron mobility transistor differential diode sensors. Sensors and Actuators B: Chemical 2008; 135: 188-194.

$11 \mathrm{Kim}$ CK, Lee JH, Lee YH et al. Hydrogen sensing characteristics of Pd-SiC Schottky diode operating at high temperature. Journal of Electronic Materials 1999; 28: 202-205.

$12 \mathrm{Kim} \mathrm{C}$, Lee J, Lee Y et al. A study on a platinum silicon carbide Schottky diode as a hydrogen gas sensor. Sensors and Actuators B: Chemical 2000; 66: 116-118.

13 Butler MA. Optical fiber hydrogen sensor. Applied Physics Letters 1984; 45: 1007-1009.

14 Butler MA. Micromirror optical-fiber hydrogen sensor. Sensors and Actuators B: Chemical 1994; 22: 155-163.

15 Liu N, Tang ML, Hentschel M et al. Nanoantenna-enhanced gas sensing in a single tailored nanofocus. Nature Materials 2011; 10: 631-636.

16 Wei X, Wei T, Xiao $\mathrm{H}$ et al. Nano-structured Pd-long period fiber gratings integrated optical sensor for hydrogen detection. Sensors and Actuators B: Chemical 2008; 134: 687-693.

17 McKeown SJ, Goddard LL. Hydrogen detection using polarization diversity via a subwavelength fiber aperture. IEEE Photonics Journal 2012; 4:, 1752-1761.

18 Raval M, McKeown S, Arbabi A et al. Palladium based Fabry-Pérot etalons for hydrogen sensing. Optical Sensors 2012, Imaging and Applied Optics Technical Papers, STh2B.5, Optical Society of America; 24-28 Jun 2012; Monterey, CA, USA; 2012.

19 Griffin BG, Arbabi A, Goddard LL. Engineering the sensitivity and response time of edge-emitting laser hydrogen sensors. IEEE Sensors Journal 2013; 13: 3098-3105.

20 Griffin BG, Arbabi A, Kasten AM et al. Hydrogen detection using a functionalized photonic crystal vertical cavity laser. IEEE Journal of Quantum Electronics 2012; 48: 160-168.

21 McKeown SJ, Goddard LL. Hydrogen detection using a single palladium nano-aperture on a fiber tip. In: Lab-on-Fiber Technology. (Cusano A, Consales M, Crescitelli A et al.) Springer International Publishing: Cham, Switzerland; 2015: 181-208.

22 Veziroğlu TN, Şahin S. 21st century's energy: Hydrogen energy system. Energy Conversion and Management 2008; 49: 1820-1831.

23 Tsukada K, Kiwa T, Yamaguchi T et al. A study of fast response characteristics for hydrogen sensing with platinum FET sensor. Sensors and Actuators B: Chemical 2006; 114: 158-163.
24 Wang M, Feng Y. Palladium-silver thin film for hydrogen sensing. Sensors and Actuators B: Chemical 2007; 123: 101-106.

25 Zhao Z, Sevryugina Y, Carpenter MA et al. All-optical hydrogen-sensing materials based on tailored palladium alloy thin films. Analytical Chemistry 2004; 76: 6321-6326.

26 Zhao Z, Knight M, Kumar S et al. Humidity effects on Pd/Au-based all-optical hydrogen sensors. Sensors and Actuators B: Chemical 2008; 129: 726-733.

27 Lee $\mathrm{E}$, Lee JM, Lee $\mathrm{E}$ et al. Hydrogen gas sensing performance of Pd-Ni alloy thin films. Thin Solid Films 2010; 519: 880-884.

28 Choi G, Jin G, Park SH et al. Material and sensing properties of Pd-deposited $\mathrm{WO}_{3}$ thin films. Journal of Nanoscience and Nanotechnology 2007; 7: 3841-3846.

29 Dai J, Yang M, Chen $Y$ et al. Side-polished fiber Bragg grating hydrogen sensor with $\mathrm{WO}_{3}$-Pd composite film as sensing materials. Optics Express 2011; 19: 6141-6148.

30 Flanagan TB, Oates WA. The palladium-hydrogen system. Annual Review of Materials Science 1991; 21: 269-304.

31 Wicke $\mathrm{E}$, Brodowsky $\mathrm{H}$, Ztichner $\mathrm{H}$. Hydrogen in palladium and palladium alloy. In: Hydrogen in Metals II, Vol. 29. (Alefeld G, Völkl J). Springer: Berlin, Heidelberg, Germany; 1978: 73-155.

32 Tabib-Azar M, Sutapun B, Petrick R. Highly sensitive hydrogen sensors using palladium coated fiber optics with exposed cores and evanescent field interactions. Sensors and Actuators B: Chemical 1999; 56: 158-163.

33 Mandelis A, Garcia JA. Pd/PVDF thin film hydrogen sensor based on laseramplitude-modulated optical-transmittance: Dependence on $\mathrm{H}_{2}$ concentration and device physics. Sensors and Actuators B: Chemical 1998; 49: 258-267.

34 Okuyama S, Mitobe Y, Okuyama K et al. Hydrogen gas sensing using a Pd-coated cantilever. Japanese Journal of Applied Physics 2000; 39: 3584-3590.

35 Baselt D, Fruhberger B, Klaassen E et al. Design and performance of a microcantileverbased hydrogen sensor. Sensors and Actuators B: Chemical 2003; 88: 120-131.

36 Henriksson J, Villanueva LG, Brugger J. Ultra-low power hydrogen sensing based on a palladium-coated nanomechanical beam resonator. Nanoscale 2012; 4: 5059-5064.

37 Edwards C, McKeown SJ, Zhou J et al. In situ measurements of the axial expansion of palladium microdisks during hydrogen exposure using diffraction phase microscopy. Optical Materials Express 2014; 4: 2559-2564.

38 Popescu G, Ikeda T, Dasari RR et al. Diffraction phase microscopy for quantifying cell structure and dynamics. Optics Letters 2006; 31: 775-777.

39 Edwards C, Arbabi A, Popescu G et al. Optically monitoring and controlling nanoscale topography during semiconductor etching. Light: Science \& Applications 2012; 1: e30.

40 Zhou R, Edwards C, Arbabi A et al. Detecting $20 \mathrm{~nm}$ wide defects in large area nanopatterns using optical interferometric microscopy. Nano Letters 2013; 13: 3716-3721.

41 Edwards C, Zhou R, Hwang SW et al. Diffraction phase microscopy: monitoring nanoscale dynamics in materials science. Applied Optics 2014; 53: G33-G43.

42 Bhaduri B, Edwards C, Pham H et al. Diffraction phase microscopy: Principles and applications in materials and life sciences. Advances in Optics and Photonics 2014; 6: 57-119.

43 Edwards C, Arbabi A, Bhaduri B et al. Measuring the nonuniform evaporation dynamics of sprayed sessile microdroplets with quantitative phase imaging. Langmuir 2015; 31: 11020-11032.

44 Shi $X$, Hesselink L. Design of a $C$ aperture to achieve $\lambda / 10$ resolution and resonant transmission. Journal of the Optical Society of America B 2004; 21: 1305-1317.

45 Coppola G, Ferraro P, lodice M et al. A digital holographic microscope for complete characterization of microelectromechanical systems. Measurement Science and Technology 2004; 15: 529-539.

46 Stoney GG. The tension of metallic films deposited by electrolysis. Proceedings of the Royal Society of London A: Mathematical, Physical and Engineering Sciences 1909; 82: 172-175.

47 Feng X, Huang Y, Rosakis AJ. On the stoney formula for a thin film/substrate system with nonuniform substrate thickness. Journal of Applied Mechanics 2007; 74: 1276-1281.

48 Hearmon R. The elastic constants of anisotropic materials II. Advances in Physics 1956; 5: 323-382.

This work is licensed under a Creative Commons Attribution 4.0 International License. The images or other third party material in this article are included in the article's Creative Commons license, unless indicated otherwise in the credit line; if the material is not included under the Creative Commons license, users will need to obtain permission from the license holder to reproduce the material. To view a copy of this license, visit http://creativecommons.org/licenses/ by/4.0/

(c) The Author(s) 2017

Supplementary Information for this article can be found on the Microsystems \& Nanoengineering website (http://www.nature.com/ micronano) 